\title{
INVARIANTS ASSOCIATED WITH SINGULARITIES OF ALGEBRAIC CURVES.
}

\author{
BY \\ TEMPLE RICE HOLLCROFT \\ of AURora, NEW York.
}

I. Introduction. Each singularity of an algebraic curre $f$, with the exception of distinct nodes, cusps, bitangents and stationary tangents, is associated with two distinct sets of invariants. ${ }^{1}$ One set, in which the number of invariants is denoted by $I_{p}$, consists of the invariants among the coefficients of the equation in point coordinates of the curve $f$; the other set, in which the number is $I_{l}$, consists of the invariants among the coefficients in the line equation of $f$. The existence of both sets of invariants is necessary and sufficient for $f$ to possess the designated singularity. Both $I_{p}$ and $I_{l}$ are independent of the order and class of $f$. The value of $I_{l}$ for any given singularity is the same as the value of $I_{p}$ for the reciprocal of this singularity.

An algebraic singularity, therefore, uniquely determines the two numbers $I_{p}$ and $I_{l}$ defined above. In this paper, the values of both $I_{p}$ and $I_{l}$ are found for a general algebraic singularity considered as defined by its constituent multiple points and their manner of combination. The chief problem is to find the value of $I_{l}$ for a singularity so defined, that is, to determine the number of invariants among the coefficients of the equation of $f$ in point coordinates associated with a general line singularity.

It has been proved by Lefschetz ${ }^{2}$ that each node of $f$ accounts for one invariant and his Postulate of Singularities states that a cusp of $f$ always accounts

"The term sinvariant" is used in this paper to mean an independent function of the coefficients of the equation of $f$ whose vanishing is necessary in order that $f$ possess a certain singularity.

$2 \mathrm{~S}$. Lefschetz, On the existence of loci with given singalarities, Transactions of the American Mathematical Society, Vol. 14 (I913), pp. 23-4I. 
for two invariants. The reciprocals of these statements will be used in studying the reciprocal singularity of a given singularity.

2. Definitions, Algebraic singularities may be divided into two general types, simple and compound. Simple point (line) singularities are built up of double points (lines) and contain no double lines (points). They are ordinarily called multiple points (lines). Compound singularities are those that contain both double points and double lines. Multiple points are formed by the coincidence of nodes and contain only those nodes which are created by the necessary crossings of the branches of the multiple point. Multiple lines are the reciprocals of multiple points.

Compound singularities result when certain multiple points of a curve become consecutive, that is, move into coincidence along given directions. The simplest example of this is the tacnode which consists of two consecutive nodes and two consecutive bitangents. Multiple points in becoming consecutive always involve the consecution of multiple lines, and conversely.

Another method of forming either simple or compound singularities, superposition, will be used in this paper. Two or more singular points are said to be superposed when they coincide so that the nature of each component singular point is not affected. The resulting singularity is the same as if the component singular points were lifted out of the plane successively and superposed without altering the relative positions of the branches within each singular point.

3. Inrariants associated with multiple points. The postulation of a simple multiple point of order $r$ is $r(v+\mathrm{I}) / 2$ and the number of invariants associated with it is $r(v+\mathrm{I}) / 2-2$. The multiple point contains $r-\mathrm{I}$ loops each of which may vanish causing a node in the singularity to be replaced by a cusp. ${ }^{1}$ For the most general multiple point of order $r$ with $k \leq r-I$ cusps

$$
I_{p}=r(r+1) / 2+k-2 .
$$

I By a vanishing loop is meant a loop that disappears becanse and only because the tangents to its two branches become coincident. Some of the loops of the curve at a singularity may be imaginary corresponding to a certain number of imaginary nodes or acnodes among the nodes composing the singularity. Since the limiting form of an acnode as the two conjugate imaginary tangents approach coincidence is a real cusp and since the tangents of a pair of im. aginary nodes may coincide respectively to form a pair of imaginary cusps, the number of invariants associated with a singularity is unaltered by the reality of its component double elements and therefore no distinction need be made as to the reality of these double elements. 
Any number $j$ of multiple points of orders $r_{i}, i=\mathrm{I}, 2, \ldots, j$, may be superposed to form a multiple point of order $\Sigma r_{i}$ which will have as many distinct tangents as the sum of the distinct tangents of the component multiple points. A multiple point thus formed contains as many cusps as the sum of the numbers occurring in the component multiple points and no more, since only nodes are added by superposition. A multiple point formed by superposition of order $r=\Sigma r_{i}$ containing $k=\Sigma k_{i}$ cusps is the same as if it had been formed by the coincidence of $r(r-1) / 2-k$ nodes and $k$ cusps for which the value of $I_{p}$ is given above.

If the $j$ multiple points are superposed in such a way that any tangent of one coincides with any tangent of another, the resulting singularity is compound and will be discussed in the next section.

4. Invariants associated with compound singularities. A compound singularity on an algebraic curve $f$ may be formed by the consecution of $s$ multiple points of $f$ each of order $r_{i}$. Assume that $r_{1} \geq r_{2} \geq r_{3} \geq \cdots \geq r_{8}$. The component multiple point of highest order $r_{1}$ is called the principal point of the singularity and the order of the principal point is the order of the resulting singularity.

Enriques ${ }^{1}$ has proved that the postulation of $s$ consecutive points of orders $r_{i}$ on $f$ is the same as the total postulation of the $s$ multiple points considered as distinct on $f$, that is

$$
\frac{\mathrm{I}}{2} \sum_{i=1}^{s} r_{i}\left(r_{i}+1\right)
$$

Assume that $s$ points are consecntive on a curve. If one of the points $P$ is fixed, one condition determines the direction of approach to $P$ of each of the remaining $s-\mathrm{I}$ points. The two parameters defining $P$ and the $s-\mathrm{I}$ parameters determining the $s-1$ directions through $P$ total $s+1$ parameters. Therefore the number of independent parameters involved in the location of $s$ consecutive points on any plane curve is $s+\mathrm{I}$.

The postulation of a singularity is the total number of conditions necessary and sufficient to determine both the nature of the singularity and its position. The $\Sigma r_{i}\left(r_{i}+\mathrm{I}\right) / 2$ relations among the coefficients of $f$ then involve the $s+\mathrm{I}$

1 F. Enriques, Lezioni sulla teoria geometrica delle equazioni e delle fanzioni algebriche, Vol. 2, pp. $404-408$. 
parameters which determine the positions of the $s$ consecutive points. From these $\Sigma r_{i}\left(r_{i}+1\right) / 2$ relations the $s+I$ parameters may be eliminated in $\Sigma r_{i}\left(r_{i}+I\right) / 2-s-I$ independent ways, each eliminant being an invariant associated with the singularity. Then for $s$ consecutive multiple points of orders $r_{i}$, there results

$$
I_{p}=\frac{\mathrm{I}}{2} \sum_{i=1}^{8} v_{i}\left(r_{i}+\mathrm{I}\right)-s-\mathrm{I} .
$$

Since a compound singularity. of order $r$ at $P$ involves $r$ branches of $f$ through $P$, these branches will form $r-$ I loops. When a loop vanishes, the node adjacent to the loop becomes a cusp. The introduction of each cusp increases by unity the value of $I_{p}$ associated with the singularity.

The most general singularity that can occur on an algebraic plane curve consists of $s$ principal $r_{1}$-fold point $P$ to which $j$ series of multiple points have become consecutive along distinct sets of branches of the principal point and, moreover, such that any point $P_{i}$ of any series may have $j_{i}$ series of multiple points consecutive along distinct sets of branches of $P_{i}$. For any singularity of this nature, the theorem of Enriques still holds.

In any such series of consecutive points, one condition is necessary and sufficient to determine the position of each point consecutive to $P$ whether along the same or distinct sets of branches. The positions of the $s$ consecutive points are, therefore, in the most general case, determined by $s+\mathrm{I}$ conditions.

The most general algebraic singularity of order $r_{1}$ contains $r_{1}$ branches and therefore $r_{1}-$ I loops any of which may vanish and introduce a cusp which replaces a constituent node.

Finally, the most general algebraic singularity of order $r_{1}$ consisting of $s$ consecutive $r_{i}$-fold points which have become consecutive in the general way described and containing $k \leq r_{1}-$ I cusps gives rise to the following number of invariants

$$
I_{p}=\frac{\mathrm{I}}{2} \Sigma r_{i}\left(r_{i}+\mathrm{I}\right)+k-s-\mathrm{I}
$$

Compound singularities are formed by superposition when multiple points are superposed so as to have a common tangent. Any number $j$ of multiple points of orders $r_{i}$ can be superposed so that all have a common tangent. The resulting singularity is of order $\Sigma r_{i}$ and consists of a principal point of order $\Sigma r_{i}$ and a consecutive $j$-fold point. The value of $I_{p}$ is obtained by regarding 
the singularity as formed by the consecution of two multiple points of orders $\mathbf{s}_{i}$ and $j$.

Any number of compound singularities may be superposed so that two or more have a common tangent which is a multiple tangent for any or all of the points concerned. As above, the value of $I_{p}$ for the resulting singularity is found by considering that singularity as formed by consecution.

A compound singularity may be a singular point some of whose tangents are multiple tangents. A tangent at a multiple point $P$ may be a multiple tangent of any order $q$ with simple contact at $P$ or with any number of points of contact up to and including $q$ located at $P$. In this case, the singularity accounts for one more invariant than the sum of the invariants belonging to the two singularities when distinct, because if either the point or line is given, the other has but one degree of freedom. The simplest example of such a singularity is the flecnode, for which $I_{p}=2$.

5. The determination of $I_{l}$ for a given singularity. The number $I_{l}$ of invariants among the coefficients of the equation of $f$ in line coordinates which express the condition that $f$ possess a given singularity is identical with the number of invariants among the coefficients of the equation of $f$ in point coordinates which express the condition that $f$ possess the reciprocal of this given singularity. This follows from the principle of duality.

Nodes and cusps do not account for invariants among the coefficients of the line equation of a curve just as bitangents and stationary tangents account for no invariants among the coefficients of its point equation.

The following theorem will be useful in determining the value of $I_{l}$ for a singularity whose point constituents are known:

Theorem I. If among the coefficients of the equation in point coordinates of an algebraic curve $f$ there exist $I_{p}{ }^{\prime}$ invariants in addition to those accounted for by the nodes and cusps of $f$, then among the coefficients of the equation of $f$ in line coordinates there must exist $I_{l}^{\prime}$ invariants in aldition to those associated with the bitangents and stationary tangents of $f$, such that $I_{p}^{\prime}=I_{l}^{\prime}$.

Consider and algebraic curve $f$ of order $n$, class $m$, with $\delta$ nodes, $x$ cusps, $\tau$ bitangents and $\iota$ inflections. Let there be $I_{p}^{\prime}$ invariants among the coefficients of the point equation of $f$ which vanish in addition to the $\delta+2 \%$ invariants due to the double points and $I_{l}^{\prime}$ invariants among the coefficients of the line equation 34-30534. Acta mathematica. 56. Imprimé le 7 novembre 1930. 
of $f$ which vanish in addition to the $\tau+2 \iota$ invariants due to the double tangents. To prove $I_{p}^{\prime}=I_{l}^{\prime}$.

Since the curve $f$ has a definite number of degrees of freedom, the equations of $f$ in point and line coordinates must contain the same number of independent coefficients. This fact is expressed by the equality

$$
\frac{\mathrm{I}}{2} n(n+3)-\delta-2 x-I_{p}^{\prime}=\frac{\mathrm{I}}{2} m(m+3)-\tau-2 \iota-I_{l}^{\prime} .
$$

From Pliicker's equations, there results for any algebraic curve

$$
\frac{1}{2} n(n+3)-\delta-2 x=\frac{1}{2} m(m+3)-\tau-2 \iota .
$$

Subtracting this relation from the one above, we obtain

$$
I_{p}^{\prime}=I_{l}^{\prime}
$$

This theorem readily determines the value of $I_{l}$ for a multiple point of order $r$ containing $k \leq r-1$ cusps. We have seen that such a singularity contains $v(r-\mathrm{I}) / 2-k$ nodes and $k$ cusps and that for it $I_{p}=r(r+\mathrm{I}) / 2+k-2$. The nodes and cusps contained in this $r$-fold point account for $r(r-\mathrm{I}) / 2+k$ invariants. Subtracting this number from $I_{p}$, we obtain $r-2$ as the number of invariants belonging to the singularity in addition to those accounted for by the nodes and cusps contained in it. Assuming that this singularity belongs to a curve that has no further point singularities or line singularities of order greater than two, apply the above theorem and there results:

For any multiple point of order $r$ containing $k \leq r-1$ cusps, that is, with or without consecutive tangents,

$$
I_{l}=r-2
$$

This theorem may also be used to determine the value of $I_{l}$ for a singularity formed by superposition. Superpose $j$ singularities, simple or compound, each occurring at a single point so that no tangent of one coincides with a tangent of another. A certain number of nodes are added by the superposition. Consider that all the $j$ singularities are possessed by the same curve and determine what part of the increase in $I_{p}$ due to superposition is over and above the increase due to added nodes. 
Let $I_{p, i}$ and $I_{l, i}$ respectively represent the numbers of invariants associated with the $j$ distinct singularities of orders $r_{i}$. Some or all of these singularities may be compound. The value of $I_{l, i}$ for a compound singularity will be obtained in the next section. Its value does not affect the determination of the additional invariants due to superposition.

When singular points are superposed, the nodes added are created by the intersections of the branches of the component singular points, each branch of each singular point intersecting every branch of every other singular point. The number of additional nodes, therefore, depends only on the orders of the component singular points. The number $d$ of additional nodes is therefore the same for $j$ multiple points of orders $r_{i}$ as for $j$ compound singular points of the same orders $r_{i}$. Then in determining $d$ for any set of superposed singular points, we need consider only the principal points of the singularities involved. The $j$ principal points of orders $r_{i}$ form a single principal point of order $\Sigma r_{i}$ which contains all of the $d$ nodes added by superposition. The number $d$ is, therefore,

$$
l=\frac{\mathrm{I}}{2} \sum_{i=1}^{j} r_{i}\left(\sum_{i=1}^{j} r_{i}-\mathrm{I}\right)-\frac{\mathrm{I}}{2} \sum_{i=1}^{j} r_{i}\left(r_{i}-\mathrm{I}\right)
$$

The various series of multiple points consecutive to the component principal points are, after superposition, consecutive to the resulting principal point along mutually exclusive branches. These consecutive multiple points occur in the same way as before superposition, since, by the definition of the process of superposition, the nature of each component singular point is not affected bj it. Therefore the number of invariants associated with the consecutive multiple points is unchanged by superposition. The difference in the number of invariants associated with the principal point of the singularity formed by superposition and the sum of the numbers associated with each of the component principal points considered as distinct is, then, the total number of invariants added by superposition. This number is

$$
\frac{\mathrm{I}}{2} \Sigma r_{i}\left(\Sigma r_{i}+\mathrm{I}\right)-2-\frac{1}{2} \Sigma r_{i}\left(r_{i}+\mathrm{I}\right)+2 j=d+2(j-\mathrm{I})
$$

This reusult shows that in addition to the $d$ invariants belonging to the additional nodes, $2(j-I)$ invariants are added by the superposition of $j$ singular points of orders $r_{i} \geq 2$. 
Therefore a singularity formed by the superposition of $j$ singular points of orders $r_{i} \geq 2$ has

$$
\begin{aligned}
& I_{p}=\Sigma I_{p, i}+d+2(j-\mathrm{I}) \\
& I_{l}=\Sigma I_{l, i}+2(j-\mathrm{I}) .
\end{aligned}
$$

The expression for $I_{l}$ results directly from Theorem I.

6. The determination of $I_{l}$ for a compound singularity. As stated in section four, the most general algebraic singularity at a single point can be formed by the consecution of multiple points. This singularity is defined by the number, orders and manner of combination of its component multiple points. For such a singularity, $I_{p}$ has been found in section four. The problem now is to determine the value of $I_{l}$ for a singularity so defined.

Let the singularity consist of a principal point $P$ of order $r$ to which $j$ series each containing $s_{i}$ multiple points are consecutive along $j$ mutually exclusive sets of branches through $P$. Any series may subdivide as described in section four. Let the point of each series adjacent to the principal point $P$ be denoted by $P_{i}$ of order $r_{i}, i=1,2, \ldots, j$.

If a series of consecutive points should subdivide at any point, this will have no effect on the order of the multiple tangent formed by that series. For example, if two or more series of multiple points are consecutive to $P_{1}$ along mutually exclusive sets of branches of $P_{1}$, this does not alter the fact that all the $r_{1}$ branches of $P_{1}$ must have contact with each other at $P$ because $P_{1}$ is consecutive to $P$ along $r_{1}$ branches of $P$. Then, whether any series of consecutive points subdivides or not, the resulting singularity contains $j$ multiple tangents of orders $r_{i}$.

Assume that between each two adjacent sets of the $j$ sets of $r_{i}$ branches there are $a_{i}$ distinct simple branches of the principal point such that $a_{i} \geq 0$. The entering and retiring branches of $f$ through $P$ may belong to sets of simple branches so there may be $j+$ I sets of branches of $f$ through $P$ each containing $a_{i}$ branches with distinct simple tangents. The order of the resulting singularity is, therefore,

$$
\sum_{i=1}^{j} r_{i}+\sum_{i=1}^{j+1} a_{i}=r
$$


The same singularity can also be formed by the superposition of $j+1$ multiple points of orders $a_{i}$ and $j$ compound singular points of orders $r_{i}$ alternately. Let the value of $I_{l}$ for each of these $j$ component compound singular points be denoted by $I_{1, i}$. Then by the method of the preceding section, by which the value of $I_{l}$ for a singularity formed by superposition was obtained, there results for the complete singularity at $P$

$$
I_{l}=\sum_{i=1}^{j}\left(I_{l, i}-r_{i}\right)+r+2(j-\mathrm{I})
$$

In the above, the computation of $I_{l}$ for the most general singularity at a single point has been reduced to the computation of the values of $I_{l, i}$ each of which is associated with a compound singularity at a single point with one and only one distinct tangent.

The following theorem will complete the process:

Theorem II. If a compound singular point has but one distinct tangent and contains neither cusps nor inflections, then for it

$$
I_{l}=I_{p}
$$

It has been proved by C. A. Scott' that if a singularity at a point has but one distinct tangent, the number of its latent nodes is equal to the number of its latent bitangents. By latent nodes [bitangents] is meant all the nodes [bitangents] contained in the singularity in addition to the $r(r-I) / 2$ nodes $[q(q-\mathrm{I}) / 2$ bitangents] necessary to form the principal point [line] of order $r[q]$ of the singularity.

Consider a compound singularity of order $r_{1}$ consisting of $s$ consecutive multiple points of orders $r_{i}$ such that $r_{1}=r_{2} \geq r_{3} \geq \cdots \geq r_{8}$. The singularity has but one tangent which has contact with $r_{1}$ branches at the singular point. This tangent is therefore of order $r_{1}$. The number of latent nodes $d^{\prime}$ and latent bitangents $t^{\prime}$ is

$$
d^{\prime}=t^{\prime}=\frac{\mathrm{I}}{2} \sum_{i=2}^{8} r_{i}\left(r_{i}-\mathrm{I}\right)
$$

1 C. A. Scott, The nature and effect of singularities of plane algebraic curves, American Journal of Mathematies, Vol. I5 (1893), p. 235. 
and the total number of nodes $d$ and bitangents $t$ contained in the singularity is, therefore,

$$
d=t=\frac{\mathrm{I}}{2} \sum_{i=1}^{*} r_{i}\left(r_{i}-\mathrm{I}\right)
$$

This singularity is assumed to contain no cusps. It will therefore contain no inflections, since only bitangents are involved in the consecution of multiple points with distinct branches. This singularity is also assumed to be built up by a single series of consecutive multiple points, but the series may subdivide at any point or points without altering the final result.

Let $I_{p}$ and $I_{l}$ be the numbers of invariants associated with this singularity. Let $n$ be the order, $m$ the class and $\tau$ and $\iota$ the total number of bitangents and inflections respectively of an algebraic curve possessing this singularity and no other except double lines. By Plücker's equations, since $d=t$,

$$
\begin{aligned}
m & =n(n-1)-2 t \\
\iota & =3 n(n-2)-6 t \\
2 \tau & =2 t+\left(n^{2}-2 n-2 t\right)\left(n^{2}-2 t-9\right) .
\end{aligned}
$$

For the reason given in Theorem $\mathrm{I}$

$$
\frac{\mathrm{I}}{2} n(n+3)-I_{p}=\frac{\mathrm{I}}{2} m(m+3)-I_{l}--(\tau-t)-2 \iota .
$$

Substitute in this equality, the values of $m, \tau$ and $\iota$ in terms of $n$ and $t$, simplify and there results

$$
I_{p}=I_{l}
$$

thereby proving the theorem.

The value of $I_{l}$ for the complete singularity has been defined by a formula which involves the numbers $I_{l, i}$. These numbers $I_{l, i}$ are associated with compound singular points each of which has but one distinct tangent. Then by Theorem II each $I_{l, i}$ may be replaced by its corresponding $I_{p, i}$ and the final formula obtained defining the value of $I_{l}$ for the complete singularity:

$$
I_{l}=\sum_{i=1}^{j}\left(I_{p, i}-r_{i}\right)+r+2(j-1)
$$

wherein 


$$
I_{p, i}=\frac{1}{2} \sum_{h=1}^{n_{i}} r_{i, h}\left(r_{i, h}+\mathrm{I}\right)-s_{i}-\mathrm{I}
$$

is the number of invariants accounted for by $s_{i}$ consecutive multiple points of orders $r_{i, h}$ where $i$ is fixed for any one series and $r_{i, 1}=r_{i, 2}$.

The value of $I_{l}$ for the most general singularity at a point $P$ is therefore given in terms of the order $r$ of the singularity, the number $j$ of distinct sets of branches along which one or more multiple points are consecutive to the principal point, the number of branches $r_{i}$ in each set and the numbers $l_{p, i}$ each of which is the number of invariants accounted for by one of the $j$ sets of $r_{i}$ branches considered as a distinct singularity.

Compound singularities formed by the superposition of singular points such that tangents coincide have been discussed in section four. In every case, the singularity could have been formed by the consecution of multiple points and the value of $I_{p}$ was so obtained. Likewise in each case the value of $I_{l}$ can be obtained by treating the singularity as if formed by consecutive multiple points.

At the end of section four, singularities that consist of singular points some of whose tangents are multiple lines were discussed. Since no double points or lines are added by the coincidence, by Theorem $I$ the values of $I_{p}$ and $I_{l}$ are each increased by the same amount which is unity for each such coincidence. For example, the value of $I_{l}$ for a flecnode is three.

7. The effect of cusps on the value of $I_{l}$. So far, in determining the value of $I_{l}$ for a compound singularity, it has been assumed that the singularity contains no cusps. The occurrence of cusps in a simple multiple point does not affect the value of $I_{l}$, but this does not hold for compound singularities.

Any singularity of order $r$ at $P$ contains $r$ branches of $f$ through $P$ and these branches form $r-I$ loops. The maximum number of cusps that may occur as components of any singularity, simple or compound, of order $r$ is, therefore, $r-I$.

In a compound singularity, both branches of certain loops have the same tangent. Such a loop must, therefore, contain a point of inflection. This loop vanishes when the node adjacent to it becomes a cusp, but when this occurs, the stationary tangent at the inflection on the loop coincides with the tangent to the singularity, replacing one of the bitangents. This occurs whenever a loop formed by branches involved in the consecution of the principal point with one 
or more multiple points vanishes. Then, since replacing a bitangent by a stationary tangent increases the value of $I_{l}$ for that singularity by unity and since this occurs when each cusp is introduced, it follows that replacing a node by a cusp in a compound singularity (except, as noted below, in that part of the principal point not involved in the consecution) increases the value of $I_{l}$ as well as that of $I_{p}$ by unity.

When a cusp is introduced in the principal point replacing a node whose branches are not involved in the consecution of the principal point with one or more multiple points, $I_{p}$ is increased by unity, but $I_{l}$ is not affected just as in the case of a simple multiple point.

Wells College, Aurora, New York, U. S. A. 